70 THE VIEWS OF MEDICAL STUDENTS AND PROFESSIONALS ON MEDICAL LEADERSHIP IN 20142015 VS 2020-2021

Jabin Chowdhury, Victor Kimani, Haroon Ali Shah. UK

10.1136/leader-2021-FMLM.70

The Birmingham Medical Leadership Society (BMLS) sought to identify whether medical students and doctors' perceptions on leadership as well as the preferred methods for teaching it, had changed.

Two identical questionnaires were compiled: one between 2014-2015 and the other between 2020-2021. Results suggest perceptions on the importance of medical leadership and management training have changed, with a statistically significant difference (95\% significance level) in results, unlikely due to chance. In 2014-2015, the largest group of participants, 17/38 $(44.7 \%)$ rated it $4 / 5$ on the Likert scale. Comparatively, in 2020-2021, 75/122 participants (61.5\%) rated it 5/5.

The value placed on medical leadership has increased, with a statistically significant difference between survey results. In 2014, 39.5\% of participants felt leadership education should be introduced in the pre-clinical years and $47.4 \%$ in clinical years. In 2020, 53.3\% of students and professionals felt this should be introduced in pre-clinical years and 38.5\% in clinical years. Current and prospective doctors desire that leadership training be introduced earlier on in one's academic career.

Accommodating this demand for earlier exposure to medical leadership and management training, may require medical schools to work with the GMC to revise medical school curricula. Delivering leadership training through NHS Paired Learning Schemes, as desired by most participants, would require cooperation from current NHS leaders to act as teacher/mentors.

The results suggest that medical leadership is seen now in higher regard than previously seen and there is a demand for early leadership training during medical school.

Earlier training may be beneficial to healthcare staff, with doctors better equipped to communicate with the multidisciplinary team, leading to improved patient outcomes. It will also provide doctors with more confidence to enter senior leadership positions.

\section{TO TRAIN AND TO SAVE: MDSSC'S EXPERIENCE ON PPE SUBSTITUTES OR SIMULATION TRAINING DURING COVID-19 OUTBREAK}

Jeff Leung-Kit Hung, Eric Hang-Kwong SO, Victor Kai-Lam Cheung, Sze-Sze SO, George Wing-Yiu NG, Nam-Hung Chia. Multi-disciplinary Simulation and Skills Centre, Queen Elizabeth Hospital, Hospital Authority, HKSAR

10.1136/leader-2021-FMLM.71

Introduction In view of global shortage of Personal Protective Equipment (PPE), Hospital Authority faced the dilemma of conserving PPE for clinical use versus consuming over 1,400 sets of PPE for simulation training in February 2020. To stockpile for an emergency preparedness, prudent use of PPE in non-emergent and non-essential services seemed necessary but not sufficient. The gist of management in healthcare simulation was the balance of achieving training objectives (trained over 1,400 staff to prevent nosocomial infection and transmission during high-risk procedures) with adequate fidelity and reducing PPE consumption for clinical use.

Methods Multi-disciplinary Simulation and Skills Centre (MDSSC) took reasonable steps to substitute 'genuine PPE' in training: i) produced 720 handcrafted face shields with kitchen sponge and laminating pouch film, ii) prototyped and printed the eye-visor frame with 3D-printing technology, iii) sourced industrial-used N95 respirators from online shop, and iv) applied expired stock/low-price gown from e-procurement for doffing of PPE practice. The extent to which reduced fidelity, followed by replacement of genuine PPE with alternative items, sacrifices overall training satisfaction was evaluated using 5-point Likert scales.

Results Training coverage for $80 \%$ healthcare professionals working closely with suspected and confirmed cases was achieved. An acceptable trade-off was identified: In sacrificing $3 \%$ of realism, no significant drop ( $p>.05)$ was found in psychological safety, mental preparedness, as well as overall satisfaction.

Discussion With the support of hospital management and administrative departments, decisive, cohesive, and sustainable responses under good leadership are keys. When it comes to extremely immense demand of PPE, innovation of intellectual minds enabled entire healthcare system to optimize effectiveness in resources allocation for quality service and safety of service users and healthcare providers.

\section{TOMORROWS' LEADERS - A LEADERSHIP AND MANAGEMENT COURSE FOR MIDDLE GRADE DOCTORS LED BY RCP CHIEF REGISTRAR}

Vaishnavi Kumar, Sarbjit Clare. City Hospital, Sandwell and West Birmingham Hospitals, UK

\subsection{6/leader-2021-FMLM.72}

Higher specialist training schemes focus heavily on gaining the necessary knowledge and skills to be clinically competent yet the opportunity to gain leadership and management experience as a trainee is comparatively limited. As RCP Chief Registrar, I designed and delivered a 3-day leadership and management programme to middle grade doctors at SWBH NHS Trust entitled 'Tomorrow's Leaders'.

'Tomorrow's Leaders' was designed to provide delegates with an introduction to NHS leadership and Management through a blended programme. The programme was delivered face-to-face with 25 middle grade doctors. The main aim was to introduce NHS leadership and management to middle grade doctors, to empower them to consider leadership and management roles earlier on in their careers and prepare them to being a consultant in the NHS. The course allowed delegates to discover their personal influencing styles and their Myers-Briggs Type Indicator. Delegates got the opportunity to meet leaders and managers within the organisation and learn about their personal journey, had formal conflict resolution training, had sessions on clinical governance, dealing with complaints, formal GMC referral pathways and coroners referrals process. The course ended with session on preparing to be a new consultant and tips on job applications and interview preparation.

Pre-course and post-course questionnaires were used. Precourse, $40 \%$ of the delegates understood our trust board structure, post-course $100 \%$ of the delegates gained this knowledge and understanding. Similarly, understanding of the 
NHS management structure improved from 33\% to $93 \%$. $100 \%$ of the delegates who attended the course found it helpful and would recommend it to a colleague. $90 \%$ of the delegates would consider taking on more leadership and management roles in the future. These findings suggest that there is a great need for such courses to narrow the leap from being a registrar to a consultant.

\section{TRAINEES TAKING CHARGE - DESIGN AND DELIVERY OF A PACES PREPARATION COURSE}

${ }^{1}$ Nora van der Stelt, ${ }^{2}$ Amy Coulden, ${ }^{3}$ Imogen Sutherland, ${ }^{4}$ Anna Naito. ${ }^{1}$ Ealing Hospital, Southall, Middlesex, UK; ${ }^{2}$ Queen Elizabeth Hospital, Birmingham, UK; ${ }^{3}$ Frimley Park Hospital, Camberley, UK; ${ }^{4}$ King's College Hospital, London, UK

\subsection{6/leader-2021-FMLM.73}

Membership of the Royal College of Physicians (MRCP) is compulsory for Internal Medical Trainees (IMTs) moving into higher specialty training. The practical component of MRCP the PACES (Practical Assessment of Clinical Examination Skills) exam - requires extensive preparation. No structured PACES teaching programme existed at St Helier Hospital, UK. In the authors' experience, local PACES preparation courses are often organised by those who themselves sat the exam many years ago, whereas trainees with recent exam experience are in an excellent position when it comes to providing preparation support.

The authors, at the time all IMTs with recent PACES experience, designed and delivered a teaching programme over a three-month period. The course consisted of weekly bedside and simulated teaching sessions tailored to candidates' specific needs, and a pan-London mock PACES exam to give candidates the opportunity to practise their skills in an environment closely resembling the real-life experience.

Direct feedback was obtained both pre and post course, and following each individual session. Questions focussed on such issues as ease of access to PACES teaching and perceived exam preparedness. Pre-course feedback indicated that $86 \%$ of candidates felt teaching was difficult to obtain, whereas post course $100 \%$ felt this was now easily available. Perceived preparedness across all stations increased from 2.3-3.9 on a 10point scale pre-course, to $7.8-8.7 / 10$ post-course.

The success of this project stressed the importance of realising that leadership is not so much a matter of seniority as it is of experience. Having only recently sat the exam themselves, the authors were in a unique position to identify the needs of PACES candidates and lead a successful preparation programme. Not only is this beneficial for exam candidates; it offers junior trainees the opportunity to develop and display leadership skills that will prove invaluable throughout the rest of their careers.

\section{USE OF SIMULATION TO DEVELOP LEADERSHIP}

Daniel Campbell. South Tyneside and Sunderland NHS Foundation Trust, UK

\subsection{6/leader-2021-FMLM.74}

Aims To investigate the impact of participation in acute simulation sessions on foundation doctors perceived confidence in their leadership abilities.
Methods Data was collected from participants who attended a foundation programme acute simulation session. They were asked to complete a pre and post session questionnaire regarding their perceived confidence in their leadership abilities, which they rated using a 5-point Likert scale. The pre and post score averages were calculated to ascertain if there was a change in the participants perceptions following the session.

Results 60 participants attended the session and completed the questionnaire. When asked about their confidence in leading whilst managing acutely unwell patients the pre-session average was 2.7 and post average score 3.8. When asked about completing an $\mathrm{ABCDE}$ assessment and making an initial management plan the pre-session average was 3.8 and post-session 4.8 , and when asked about their confidence in recognising the limitations of their abilities the pre-session average was 4.2 and the post-session average 4.6.

Conclusions The results of this project have highlighted the low confidence foundation doctors perceived in their abilities to lead in acute situations. Conversely, they felt confident in recognising situations where they had reached the limitation of their abilities. There was a positive outcome from attending the session with an increase of 1.1 in the average score concerning participants confidence in leading acute situations and 1.0 in performing an ABCDE assessment and making an initial management plan. Overall, this work has demonstrated that attendance at an acute simulation session has improved the foundation doctor's confidence in their leadership abilities. Further work is needed to ascertain what factors influence junior doctor's perceptions of their leadership skills and whether the reported improvement in confidence impacted their clinical practice.

\section{USING A BLENDED LEARNING APPROACH TO DEVELOP QUALITY IMPROVEMENT SKILLS AMONGST JUNIOR DOCTORS}

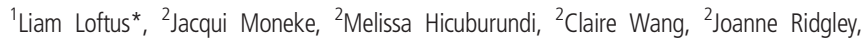
${ }^{2}$ Rebecca Bennewith, ${ }^{2}$ Natasha Aghtarafi. ' GP Trainee, Cambridge, and Health Education East of England Leadership Fellow; ${ }^{2}$ Foundation Year Doctor, Cambridge University Hospital

\subsection{6/leader-2021-FMLM.75}

Aims Leadership competencies and quality improvement skills are becoming an increasingly important element of junior doctor training curricula. How we support our junior doctors to develop these skills must therefore advance to match this.

This work aimed to take a blended learning approach, using eLearning modules alongside a real-world project, to develop quality improvement (QI) capability amongst Foundation Year (FY) doctors.

Methods Four eLearning modules were created by Health Education East of England Leadership Fellows, which covered all the core components of completing a quality improvement project (QIP). A Leadership Fellow then engaged six FY doctors, who collaborated on four QIPs, which aimed to increase the number of Fit Notes issued to patients upon discharge from a large teaching hospital.

They received direction from the eLearning modules, in addition to light-touch supervision from a Leadership Fellow (specifically an initial meeting, 4-6 weekly catch-up emails, and ad-hoc troubleshooting). 